\title{
BROADENING THE SCOPE OF THE EASTERN SYDNEY AREA SENTINEL SURVEILLANCE NETWORK
}

Leena Gupta and Mark Ferson

Eastern Sydney Public Health Unit

$T^{1}$ he general practice-based sentinel surveillance network in Eastern Sydney Area (ESA) has been operating for two years. Its purpose has been to document temporal variations in general practice consultation rates for specific diseases, to detect epidemics or disease clusters and to contribute to Statewide influenza surveillance. Until October 1993 conditions reported by participating general practitioners (GPs) included influenza, asthma, chickenpox, hand, foot and mouth disease and shingles. Aggregate data are reported in a monthly bulletin produced by the ESA Public Health Unit.

In October 1993 we conducted a review of general practicebased sentinel surveillance in ESA. We interviewed participating GPs and contacted other units in NSW which coordinate general practice-based sentinel surveillance. The 13 GPs interviewed reported they found participation in the network useful to their clinical practice, particularly in the provision of aggregate information to patients. Several GPs considered it unnecessary to report some infectious conditions and suggested alternatives, such as domestic violence, injuries, acne in adolescence, pap smears and requests to screen for sexually transmitted diseases. In contacting other units which co-ordinate sentinel surveillance, we found that some units had broadened the scope of reporting to include such conditions as occupational injury, alcohol-related consultations and marital disharmony.

As a result of the review we modified the list of conditions reported and have now included only those in which clinical, public health or other (social, economic) intervention may be implemented in ESA, or where the impact of intervention could be monitored. Specifically, we have reduced the extent to which infectious diseases are reported and begun reporting of known or suspected cases of domestic violence. In ESA sentinel surveillance age/sex data on domestic violence have been collected for 10 months using a case definition developed with the ESA Women's Health Coordinator. GPs also classify the manifestations of domestic violence as physical, psychological, sexual, social/cultural or economic.

The data on domestic violence have considerable public health significance, in view of a previous study in an Eastern Sydney emergency department, where domestic violence accounted for 12 per cent of 512 presentations for injuries due to violence in a six-month period ${ }^{1}$. Studies elsewhere have demonstrated that between 1 per cent and 20 per cent of women who present to a hospital emergency department have a recent history of domestic violence $e^{2,3,4}$.

The collection of this information is relevant to the development and implementation of the NSW Domestic Violence Strategic Plan ${ }^{5}$, the NSW Health Department Domestic Violence Policy ${ }^{6}$ and the ESA Health Service Domestic Violence Policy and Protocol ${ }^{7}$, all of which identified the need for further data collection.

Care has been exercised in interpreting data obtained because of methodological difficulties inherent to voluntary small-scale sentinel surveillance systems. Bias is of particular concern because the surveillance network is not population-based - the participating GPs are volunteers and are not distributed evenly by geographic location, practice demographics or casemix. In addition, compliance is variable. As a result, data are not representative of the population seen by GPs in ESA. Poor sensitivity is also a significant limitation, as case definitions for domestic violence and its manifestations have been simplified to assist in rapid assessment and classification of patients for reporting purposes. This has resulted in underreporting of cases and may result in misclassification.

Despite these limitations we believe reporting of domestic violence has been valuable because of the paucity of data about the problem. From data collected by the surveillance network it will, at least, be possible to determine baseline consultation rates for participating practitioners and to detect temporal trends in consultation rates over longer periods, as further strategies to increase recognition and improve management of domestic violence are implemented. The value of the data may also be in estimating the proportion of domestic violence presentations to GPs with a certain manifestation - for example, the proportion of consultations for domestic violence in which social/cultural abuse is a manifestation. Most important, the collection of primary care data on domestic violence will complement data from, for example, emergency presentations and will be an indicator of the need for more extensive data collection.

Since ESA PHU began data collection on domestic violence, some other sentinel networks have started collecting similar data. We have also considered monitoring other conditions, to complement current clinical or preventative initiatives. These "conditions" include injuries due to falls (the leading cause of hospitalisation for injury in 1989-1990 ), presentations with scalds and referrals for mammography. The possibility of reporting self-inflicted injuries provides a mechanism by which the general practice-based sentinel surveillance network could link with the ESA Health Outcomes Council which is focusing on suicide. Amendments to the existing list of conditions will be made in consultation with participating GPs.

Previous articles in the NSW Public Health Bulletin have indicated potential benefits of general practice-based surveillance networks ${ }^{9,10}$. Mira et a $1^{10}$ have suggested that sentinel surveillance has the potential to monitor conditions other than infectious diseases and preventative care. We consider that the scope of "conditions" reported by general practice-based sentinel surveillance networks can be broadened further to provide a useful mechanism for monitoring the impact of a wide range of public health and health promotion initiatives, social welfare programs or clinical services, at a local or statewide level.

\section{ACKNOWLEDGMENT}

We gratefully acknowledge Susan Furber, David Lyle, Karen Alexander and Michael Mira for their input and thank all the general practitioners who have participated in the network.

1. Fulde GWO, Cuthbert M, Kelly R. Violence in society: fact or fiction? Emergency Med 1991; 3:37(80):51-54.

2. Roberts GL, OToole BI, Lawrence JM et al. Domestic violence victims in a hospital emergency department. Med $J$ Aust 1993; 159:307-310. 


\section{Tobacco advertising}

$\checkmark$ Continued from page 120

displayed more than one advertising sign that breached the Act.

\section{DISCUSSION}

Overall, 90.5 per cent of tobacco retailers in suburban shopping centres and 96.2 per cent of service stations complied with the Act at the initial survey. This may have been due to a number of factors. In mid-1993, the NSW Health Department wrote to all tobacco retailers explaining the legislation. In addition, before the September 30 deadline, tobacco companies began supplying legal advertising material to retailers. Some advice and advertising material from the tobacco companies, however, does not comply with the Act.

The most common advertising breaches were those that advertised the availability of tobacco products, e.g. "Cigarettes sold here". In many instances these signs were provided by the tobacco companies and were in the colours of major tobacco companies. Tobacco retailers were happy to remove these illegal advertising signs, especially when they were told they were liable to prosecution rather than the tobacco companies.

All but one tobacco retailer had complied by the final inspection. This retailer is a member of a major supermarket chain. We were told by the management that its advertising policy was determined at the corporate level and that it would eventually comply. We did not prosecute the retailer as the NSW Health Department is negotiating with the supermarket chain.

Warning letters seemed to alter advertising behaviour in some of the retailers. We did not have sufficient statistical power to evaluate the effectiveness of warning letters in changing advertising behaviour. In view of limited resources, it is important, in a future study, to compare the relative effectiveness of warning letters and visits by an EHO in changing advertising behaviour. It should be noted that very high compliance rates were achieved in the survey population without recourse to prosecutions.

Specialist tobacconists had the highest failure rate. It may be that as the retailing of tobacco products is their sole source of livelihood they are willing to risk illegal advertising. In any education campaign, they should be given a high priority.

We did not record any breaches in business names. At the time of the study, there was debate about whether it was legal to use the word "tobacconist" in registered business names. If business names such as "discount tobacconist" were not permissible, there would have been 25 additional breaches and 16 additional offending retail outlets. It is now NSW Health Department policy, however, that business signs with the word "tobacconist" are acceptable provided they are part of a registered business name and comply with the spirit of the Act.

The survey method was labour-intensive. A list of licensed tobacco retail outlets was unavailable. Such a list would have ensured a complete sampling frame.

In summary, it was gratifying that most tobacco retailers in shopping centres and service stations in western Sydney were complying with the Tobacco Advertising Prohibition Act 1991. Specialist tobacconists, however, have a high noncompliance rate. This group of retailers will need to be targeted in any follow-up publicity or education campaigns.

\section{ACKNOWLEDGMENTS}

We would like to thank the Drug and Alcohol Directorate, NSW Health Department, for funding this study.

1. US Department of Health and Services. The Health Benefits of Smoking Cessation. A Report of the Surgeon General 1990.

2. d'Espaignet ET, van Ommeren M, Taylor F, Briscoe N, Pentony P. Trends in Australian Mortality 1921-1988. Australian Institute of Health. Australian Government Publishing Service, Canberra 1990. 3. Jalaludin B, Smith W, Salkeld G, Chey T, Capon A. Population Attributable Risks in Setting Priorities for Interventions in Cancer Attributable Risks in Setting Priorities for Intervention

Prevention. Western Sector Public Health Unit 1993. 4. Dobson E, Woodward S, Leeder S. Tobacco smoking in respons
cigarette advertising. (Letter) Med J Aust 1992; 156(11):815-16. 5. Pierce JP, Gilpin E, Burns DM et al. Does tobacco advertising target young people to start smoking? Evidence from California. JAMA 1991; 266(22):3185-86.

\section{Sentinel surveillance network}

\section{$\checkmark$ Continued from page 121}

3. McLeer VA, Anwar R. A study of battered women presenting in an emergency department. Am J Public Health 1989; 79(1)65-66. 4. Goldberg WG, Tomlanovich MC. Domestic violence victims in an emergency department. JAMA 1984; 251:3259-3264.

5. NSW Domestic Violence Committee. Report of the NSW Domestic Violence Committee. NSW Domestic Violence Strategic Plan. NSW Women's Coordination Unit, July 1991.

6. NSW Health Department. Domestic Violence Policy. March 1993. 7. Eastern Sydney Area Health Service. Draft Domestic Violence Policy and Protocol. January 1994.

8. Brown JA, Towler BP, Stokes ML. Injury Profile. An overview of injury in Eastern Sydney. 1993. Public Health Unit, Eastern Sydney Area Health Service.

9. Jeffs D, McMahon R. Sentinel General Practices. NSW Public Health 9. Jeffs D, McMahon R. Se
Bulletin 1990; 12(1):51-52.

10. Mira M, Cooper C, Britt H. Benefits of general practice sentinel surveillance networks. NSW Public Health Bulletin 1992; 3(11):121-122.

\section{PUBLIC HEALTH EDITORIAL STAFF}

The editor of the Public Health Bulletin is Dr Michael Frommer, Director, Research and Development, NSW Health Department; production manager is Dr Marie-Louise Stokes, and assistant editor is Dr Valerie Delpech.

The Bulletin aims to provide its readers with population health data and information to motivate effective public health action. Articles, news and comments should be 1,000 words or less in length and include a summary of the key points to be made in the first paragraph.

Please submit items in hard copy and on diskette, preferably using WordPerfect 5.1, to the editor, NSW Public Health Bulletin, Locked Mail Bag 961, North Sydney 2059. Facsimile (02) 3919232. Please contact your local Public Health Unit to obtain copies of the NSW Public Health Bulletin. 